UCRHEP-T203

\title{
Enhanced Three-Body Decay of the Charged Higgs Boson
}

\author{
Ernest Ma ${ }^{1}$, D.P Roy ${ }^{1,2}$, and José Wudka ${ }^{1}$ \\ ${ }^{1}$ Department of Physics, University of California. Riverside, California, 92521-0413 \\ ${ }^{2}$ Tata Institute of Fundamental Research, Mumbai 400 005, India
}

(August 2, 2021)

\begin{abstract}
If the charged Higgs boson $H^{+}$exists with $m_{H^{+}}<m_{t}+m_{b}$, the conventional expectation is that it will decay dominantly into $c \bar{s}$ and $\tau^{+} \nu_{\tau}$. However, the three-body decay mode $H^{+} \rightarrow W^{+} b \bar{b}$ is also present and we show that it becomes very important in the low $\tan \beta$ region for $m_{H^{+}} \gtrsim 140 \mathrm{GeV}$. We then explore its phenomenological implications for the charged-Higgs-boson search in top-quark decay.
\end{abstract}

The discovery of the top quark at the Tevatron collider [1].2] has generated a good deal of current interest in the search for new particles in the decay of the top-quark. In particular, top quark decay is known to be a promising reaction to look for the charged Higgs boson of a two-scalar doublet model and, in particular, the minimal supersymmetric standard model (MSSM) [3]. In the diagonal CKM matrix approximation the MSSM charged Higgs boson couplings to the fermions are given by

$$
\mathcal{L}=\frac{g}{\sqrt{2} m_{W}} H^{+}\left[\cot \beta m_{u i} \bar{u}_{i} d_{i L}+\tan \beta m_{d i} \bar{u}_{i} d_{i R}+\tan \beta m_{\ell i} \bar{\nu}_{i} \ell_{i R}\right]+\text { h.c. }
$$

where $\tan \beta$ is the ratio of the vacuum expectation values of the two scalar doublets and the index $i$ labels the quark and lepton generation. This interaction implies a large $H^{+} t b$ Yukawa coupling when 


$$
\tan \beta \lesssim 1 \text { and } \tan \beta \gtrsim m_{t} / m_{b}
$$

where one expects a large branching fraction for $t \rightarrow b H^{+}$decay (given $m_{t}>m_{H^{+}}$). Interestingly the regions $\tan \beta \sim 1$ and $\gtrsim m_{t} / m_{b}$ are favored by SUSY-GUT models for a related reason - i.e. the unification of the $b$ and $\tau$ masses which requires a large negative contribution from the top Yukawa coupling to the renormalization group equation [4].

It should be noted here that the perturbation theory limit on the $H^{+} t b$ Yukawa coupling requires

$$
0.2<\tan \beta<100
$$

while the GUT scale unification constraint implies stricter limits

$$
1 \leq \tan \beta \leq m_{t} / m_{b}
$$

which are also required if one assumes the perturbation theory limit on the Yukawa coupling to remain valid up to the GUT scale [5]. Without any GUT scale Ansatz, however, the allowed region of $\tan \beta$ extends down to 0.2 . We shall assume only the particle content of the MSSM Higgs sector but no constraints from GUT scale physics. Our analysis will remain valid in any two-Higgs doublet model satisfying the coupling pattern of the MSSM as given by (1); i.e. the so-called class II models [6].

For $m_{t}>m_{H^{+}}$the dominant decay modes are usually assumed to be the two body decays $H^{+} \rightarrow c \bar{s}, \tau^{+} \nu$. The corresponding widths are

$$
\begin{aligned}
\Gamma_{c s} & =\frac{3 g^{2} m_{H^{+}}}{32 \pi m_{W}^{2}}\left(m_{c}^{2} \cot ^{2} \beta+m_{s}^{2} \tan ^{2} \beta\right) \\
\Gamma_{\tau \nu} & =\frac{g^{2} m_{H^{+}}}{32 \pi m_{W}^{2}} m_{\tau}^{2} \tan ^{2} \beta
\end{aligned}
$$

The leading QCD correction is taken into account by substituting the quark mass parameters for eqs. (11) and (5) by the running masses at the $H^{+}$mass scale. Its most important effect is to reduce the charm quark mass $m_{c}$ from $1.5 \mathrm{GeV}$ to $1 \mathrm{GeV}$ [7]. Consequently the two rates are approximately equal when $\tan \beta \sim 1$; the $\tau \nu(c s)$ rate dominates when $\tan \beta>1$ $(\tan \beta<1)$. 
In this note we shall consider the phenomenological implications of a very important 3-body decay channel of the Higgs boson, namely

$$
H^{+} \rightarrow \bar{b} b W^{+},
$$

where the $b W^{+}$comes from a virtual $t$ quark [8]. The dominant contribution comes from the top-quark exchange with a large Yukawa coupling of $\mathrm{H}^{+}$to the top quark given by the first term in eq. (1). One can easily calculate the corresponding width as

$$
\frac{d \Gamma_{\bar{b} b W}}{d s_{\bar{b}} d s_{b}}=\frac{1}{256 \pi^{3} m_{H^{+}}^{3}}\left(\frac{3 g^{4} m_{t}^{4} \cot ^{2} \beta}{4 m_{W}^{4}\left(m_{t}^{2}-s_{\bar{b}}\right)^{2}}\right)\left[m_{W}^{2}\left(s_{W}-2 m_{b}^{2}\right)+\left(s_{\bar{b}}-m_{b}^{2}-m_{W}^{2}\right)\left(s_{b}-m_{b}^{2}-m_{W}^{2}\right)\right]
$$

where $s_{\bar{b}}, s_{b}$ and $s_{W}$ are the 4-momentum squared transferred to the corresponding particles satisfying $s_{\bar{b}}+s_{b}+s_{W}=m_{H^{+}}{ }^{2}+m_{W}^{2}+2 m_{b}^{2}[9]$.

Figure 1 compares the 3 body decay width $\Gamma_{\bar{b} b W}$ with the 2 body widths $\Gamma_{c s}$ and $\Gamma_{\tau \nu}$ over the charged Higgs boson mass range $120-170 \mathrm{GeV}$ at $\tan \beta=1 . \Gamma_{\bar{b} b W}$ is seen to be the dominant decay width for $m_{H^{+}} \gtrsim 140 \mathrm{GeV}$, while the 2 body decays dominate up to $m_{H^{+}}=130 \mathrm{GeV}$. The reason for this is the large $H^{+}$Yukawa coupling to $t \bar{b}$, which is about 100 time larger than those to the $c \bar{s}$ and $\tau^{+} \nu$ channels. This can overcome the extra suppression factors due to the gauge coupling of the $W$ as well as the 3 body phase space, provided the off-shell propagator suppression factor is not too large. The latter is ensured for $m_{H^{+}} \gtrsim 140 \mathrm{GeV}$. Thus the 3 body decay (7) is the dominant mode for

$$
m_{H^{+}} \gtrsim 140 \mathrm{GeV} \text { and } \tan \beta \lesssim 1
$$

while the $\tau \nu$ mode (6) dominates at larger $\tan \beta$. The $c \bar{s}$ mode is relatively small at all $\tan \beta$ for $m_{H^{+}} \gtrsim 140 \mathrm{GeV}$. It may be noted here that the relative size of the $H^{+}$decay widths at $\tan \beta=1$ (Fig. [) would hold for all values of $\tan \beta$ in the two-Higgs doublet model of type I [6].

This situation has a close parallel in the neutral scalar sector. For a neutral Higgs $H^{0}$ whose mass is slightly below the $W W$ threshold a good detection channel is $W W^{*}$ with 
$W^{*} \rightarrow \ell \nu$. In this case the decay $H^{0} \rightarrow W \ell \nu$ is comparable to $H^{0} \rightarrow \bar{b} b[6]$. A related decay $H^{+} \rightarrow W^{+} Z^{*}$ with $Z^{*} \rightarrow b \bar{b}$ is not considered because for multi-doublet models there is no $H^{+} W^{-} Z$ coupling 10$]$.

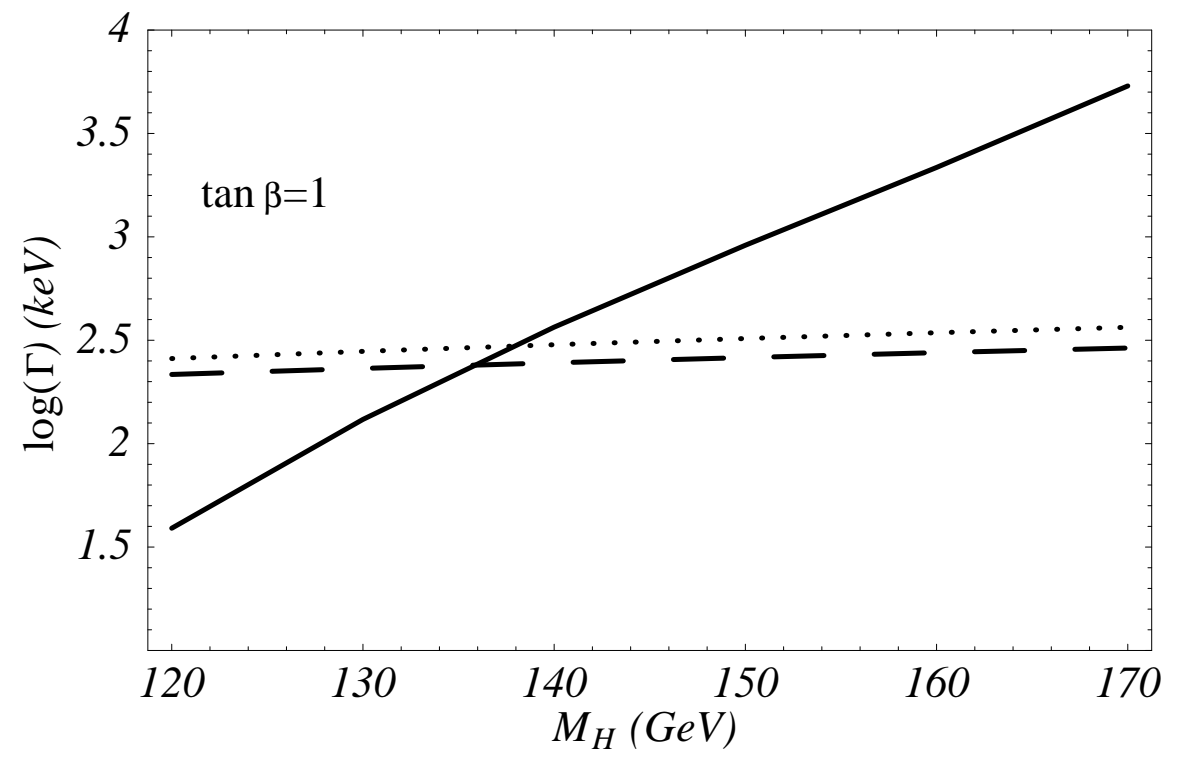

FIG. 1. Comparison of the 3 body decay width $\Gamma_{H^{+} \rightarrow \bar{b} b W}$ (solid) with the 2 body widths $\Gamma_{H^{+} \rightarrow c \bar{s}}$ (dashed) and $\Gamma_{H^{+} \rightarrow \tau^{+} \nu}($ dots $)$.

The $H^{ \pm}$search strategies in top quark decay have so far been based on the distinctive features of the channels

$$
\begin{aligned}
& t \rightarrow b H^{+} \rightarrow b \tau^{+} \nu \\
& t \rightarrow b H^{+} \rightarrow b c \bar{s}
\end{aligned}
$$

vis-a-vis the standard model decay

$$
t \rightarrow b W^{+} \rightarrow b\left(\ell \nu, \tau \nu, q^{\prime} \bar{q}\right)
$$

As we have seen above, however, this strategy is valid only up to $m_{H^{+}} \simeq 130 \mathrm{GeV}$. For $m_{H^{+}} \gtrsim 140 \mathrm{GeV}$ the $c \bar{s}$ mode (11) is overtaken by

$$
t \rightarrow b H^{+} \rightarrow b \bar{b} b W^{+} \rightarrow b \bar{b} b\left(l \nu, \tau \nu, q^{\prime} \bar{q}\right)
$$


as the dominant decay mode for the low $\tan \beta(\lesssim 1)$ region. The distinctive feature of this new channel is evidently very different from those of the channels (10) and ( 11).

In order to assess the impact of the new channel (13) let us summarize the main features of the current $H^{+}$search program in $t \bar{t}$ decay. It is based on two strategies - i) excess of $t \bar{t}$ events in the $\tau$ channel, and ii) their deficit in the leptonic $(\ell=e, \mu)$ channel with respect to the standard model prediction from (12). The first is appropriate for the large $\tan \beta$ region where the $\tau \nu$ channel (10) is the dominant channel of the charged Higgs decay. One can already get significant limits on $m_{H^{+}}$for very large $\tan \beta\left(\gtrsim m_{t} / m_{b}\right)$ from the CDF $t \bar{t}$ data in the $\ell \tau$ and inclusive $\tau$ channels [11, 12]. This analysis can be extended down to lower values of $\tan \beta$ at the Tevatron upgrade and the LHC by exploiting the opposite states of $\tau$ polarization from $W^{ \pm}$and $H^{ \pm}$decays [13]. Evidently this type of analysis would not be affected by the new channel.

The second strategy is based on a suppression of the leptonic $(e, \mu)$ decay of the top due to the $H^{+}$channels (10) and (11) t. The experimental estimate of the $t \bar{t}$ cross section is based on the $\ell \ell$ and $\ell+$ multijet channels with a $b-$ tag, requiring leptonic decay of at least one of the top quarks. Thus the presence of the $H^{+}$channels (10) and (11) would imply a decrease of this $t \bar{t}$ cross section, while the experimental estimate [14]

$$
\sigma_{t \bar{t}}(\mathrm{CDF}+\mathrm{D} \varnothing)=6.5_{-1.2}^{+1.3} \mathrm{pb} \quad \sigma_{t \bar{t}}(\mathrm{CDF})=7.6_{-1.5}^{+1.8} \mathrm{pb}
$$

is actually slightly higher than the QCD prediction of $\sigma_{t \bar{t}} \leq 5.6 \mathrm{pb}$ [15]. This has lead to a significant lower limit on $m_{H^{+}}$at low $\tan \beta(\lesssim 1)$, assuming dominance of the cs decay channel (11) [16, 17]. Evidently this method will be valid only up to $m_{H^{+}}=130 \mathrm{GeV}$. Beyond this value the dominant charged Higgs decay channel in the low $\tan \beta(\lesssim 1)$ region is (13), which does not imply any reduction in the leptonic decay of the top. Instead it implies

\footnotetext{
${ }^{1}$ This is evident for the $c s$ channel (11) but should also hold for the $\tau \nu$ channel (10) as well since the $e, \mu$ from $\tau$ decay are expected to be soft and hence suppressed by the $p_{T}$ cut used in the analysis.
} 
an increase in the $b$-tagging efficiency due to the multi- $b$ final state. Since the CDF cross section is largely based on the $b$-tagged events, the presence of the decay channel (13) would imply an increase of this cross section relative to the standard model prediction, instead of a decrease. Thus it will go in the same direction as the data.

Let us now look at the implications of the new $H^{ \pm}$decay channel (13) on Tevatron $t \bar{t}$ events more closely. In Fig. 目 we show the branching fractions for $t \rightarrow b H^{+}$and $H^{+} \rightarrow \bar{b} b W$ decays over the low $\tan \beta$ region for $m_{H^{+}}=140$ and $150 \mathrm{GeV}$. Also shown is the product of these two branching fractions,

$$
B=B(t \rightarrow b \bar{b} b W)=B\left(t \rightarrow b H^{+}\right) B\left(H^{+} \rightarrow \bar{b} b W\right)
$$

which is about the same for both values of $m_{H^{+}}$. We see that this branching fraction lies in the range $5-20 \%$ for $\tan \beta=1-0.6$. This corresponds to a probability of about $10-40 \%$ $(\simeq 2 B)$ for the channel

$$
\bar{t} t \rightarrow \bar{b} b \bar{b} b W W
$$

where one of the top-quarks decays via an $H^{ \pm}$and we have made a first-order approximation in $B$. Thus the $2 b$ and $4 b$ final states occur with relative probabilities $1-2 B$ and $2 B$ respectively, where the former also includes a small contribution from the 2-body decays of the $H^{ \pm}$.

It should be mentioned here that the decay of the $H^{ \pm}$into a neutral Higgs and a real or virtual $W$ boson is (whenever kinematically allowed) an additional source for a $4 b$ final state such as $(\sqrt{16})$. Within the MSSM this contribution can be significant over the low $\tan \beta$ region [8] depending on the SUSY breaking parameters. Thus the 3-body decay considered above constitutes a minimal contribution to the $4 b$ final state (16) generated by the decays of the charged Higgs boson.

We have studied the characteristic features of the above channel versus the standard model decay

$$
\bar{t} t \rightarrow \bar{b} b W W
$$




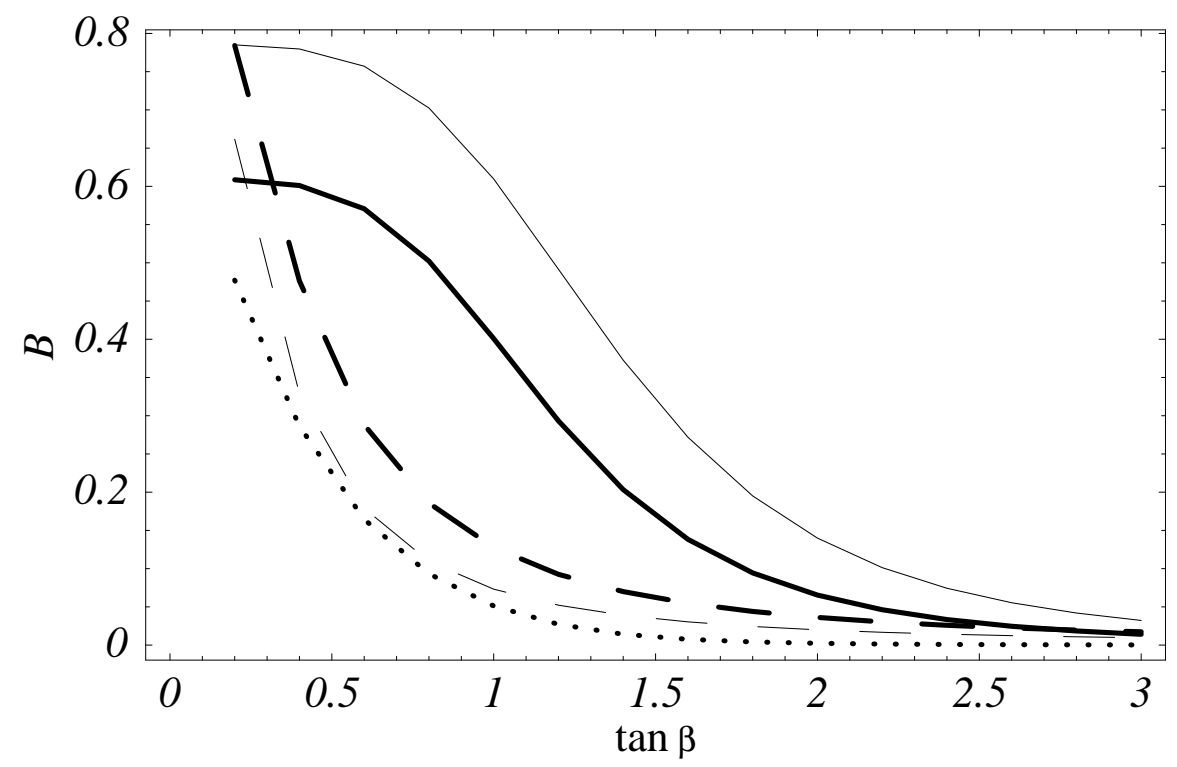

FIG. 2. Branching fractions for $t \rightarrow b H^{+}$(dashed lines) and $H^{+} \rightarrow \bar{b} b W$ (solid lines) decays for low $\tan \beta$. Heavy lines and thin lines correspond to $m_{H^{+}}=140$ and $m_{H^{+}}=150 \mathrm{GeV}$ respectively. The dotted line corresponds to the product $B\left(t \rightarrow b H^{+}\right) B\left(H^{+} \rightarrow \bar{b} b W\right)$ for $m_{H^{+}}=140 \mathrm{GeV}$ (the plot for $m_{H^{+}}=150 \mathrm{GeV}$ is practically identical).

via a parton-level Monte Carlo program. While the $\ell$ and $\nu$ from $W$ decay have very similar kinematic distributions in the two cases, there is a clear difference in the number of tagable $b$-quarks. The CDF SVX detector has a tagging efficiency of $\epsilon_{b}=0.24$ per $b$ satisfying

$$
E_{T}^{b}>20 \mathrm{GeV}, \quad\left|\eta_{b}\right|<2
$$

which takes into account the loss of efficiency due to the limited rapidity coverage of the vertex detector $\left(\left|\eta_{\mathrm{SVx}}\right| \lesssim 1\right)$ 18]. This is expected to go up to $\epsilon_{b}=0.4$ per $b$ for run II as the rapidity coverage of the vertex detector is extended to $\left|\eta_{\mathrm{Svx}}\right|=2$. Table 1 shows the probability distribution of the numbers of $b$ quarks per event satisfying the tagging criterion (18) for the signal (16) and the standard model background (17) channels. It shows that the majority of the signal events are expected to contain 3-4 tagable $b$ quarks for $m_{H^{+}}=140 \mathrm{GeV}$ ( similar results hold for $m_{H^{+}}=150 \mathrm{GeV}$ ). It also shows the probability distribution for the expected number of $b$-tags per event for the SVX tagging efficiency of $\epsilon_{b}=0.24$, where we 
have assumed that the uncorrelated probability for tagging $n$ out of $N$ tagable $b$-quarks is $P_{n}^{N}=\left(\begin{array}{l}N \\ n\end{array}\right) \epsilon_{b}^{n}\left(1-\epsilon_{b}\right)^{N-n}$. The corresponding expectations for the run II efficiency $\epsilon_{b}=0.4$ are shown in parenthesis. The implications for the $t \bar{t}$ events in the $b$-tagged $\ell+$ multijet channel are discussed below.

\begin{tabular}{|c||c|c|c|c||c|c|c|}
\hline \multicolumn{1}{|c||}{ Probability (\%) } & \multicolumn{3}{c||}{ No. of tagable $b$ 's/event } & \multicolumn{4}{c|}{ No. of $b$-tags/event } \\
& 1 & 2 & 3 & 4 & $\geq 1$ & $\geq 2$ & $\geq 3$ \\
\hline $\bar{t} t \rightarrow \bar{b} b \bar{b} b W W(2 B)$ & 4.7 & 25.6 & 50.6 & 18.9 & $52.8(74.2)$ & $12.4(31.8)$ & $(6.6)$ \\
\hline $\bar{t} t \rightarrow \bar{b} b W W(1-2 B)$ & 13 & 87 & - & - & $39.6(60.9)$ & $5(13.4)$ & - \\
\hline
\end{tabular}

Table 1. Probabilities for different numbers of tagable $b$ quarks per event and numbers of $b$-tags (per event) with $\epsilon_{b}=0.24(0.4)$ for the $H^{ \pm}$signal $\left(m_{H^{+}}=140 \mathrm{GeV}\right)$ and the standard model background.

As we see from this table the probability of inclusive single $(\geq 1)$ b-tag is $52.8 \%$ for the signal compared to $39.6 \%$ for the standard model decay, i.e., about 1/3 higher. Consequently the measured $\bar{t} t$ cross section will appear larger than the standard model prediction by $(1 / 3) \times(2 B)$, i.e. about $13 \%$ for $B=0.2$. This could account for at least part of the excess of the CDF $t \bar{t}$ cross section [14] over the standard model prediction. Even more significantly, the probability for inclusive double $(\geq 2)$ b-tag is $12.4 \%$ for the signal compared to only $5 \%$ for the standard model decay, i.e. an excess of $150 \%$. This would imply an excess of double $b$ tagged events over the standard model prediction by $3 B$, i.e. $60 \%$ for $B=0.2$. Again there seems to be an indication of such an excess in the CDF data [19]. It should be remarked however that the excess is expected to appear in the $\geq 3$ jet events; but not in the 2 jet sample, except through fluctuations. It is therefore premature to link the reported excess to the above mechanism. It is important to note, however, that the size of the signal can have visible impact even at the level of the existing limited data.

It should be noted here that one expects a 20 -fold rise in the number of $t \bar{t}$ events in the run II, and the efficiency of single and double tags to go up by factor of 1.5 and $\sim 3$ respectively. Thus one expects about 1000 single $(\geq 1)$ and 200 double $(\geq 2) b$-tagged events for CDF, and similar numbers for DØ (in run II). Even with a $B$ of only $5 \%$, this would 
correspond to an excess of $\sim 30$ double $b$-tagged events, i.e. a $2-3 \sigma$ effect. Moreover, the $6.6 \%$ efficiency for $\geq 3 b$-tags for the signal would imply at least $10-12$ triple $b$-tagged events for $B \geq 5 \%$. Finally, one should be able to get additional constraints from the clustering of the reconstructed $H^{ \pm}$mass.

Thus the 3-body decay channel provides a visible signature for a charged Higgs boson in top-quark decay over its region of dominance, i.e. $m_{H^{+}} \gtrsim 140 \mathrm{GeV}$ and $\tan \beta \lesssim 1$. This can be used to probe for an $H^{ \pm}$at the Tevatron run II over the mass range $140-150 \mathrm{GeV}$, and can be extended beyond $160 \mathrm{GeV}$ at the LHC. We conclude with the hope that this channel will play an important role in the charged Higgs boson search program in the future.

\section{ACKNOWLEDGMENTS}

We thank Prof. V. Barger for discussions and Dr. M. Mangano and Dr. G.P. Yeh for several communications regarding the CDF $b$ tagging efficiency. This work was supported in part by the Department of Energy under Grant DE-FG03-94ER40837. 


\section{REFERENCES}

[1] F. Abe et al. (CDF Collaboration), Phys. Rev. Lett. 74, 2626 (1995). S. Abachi et al. (DØ Collaboration), Phys. Rev. Lett. 74, 2632 (1995).

[2] P. Tipton, proceedings of the 28th Int. Conf. on High Energy Physics, Warsaw (1996).

[3] V. Barger and R.J.N. Phillips, Phys. Rev. D41, 884 (1990). A.C. Bawa, C.S. Kim and A.D. Martin, Z. Phys. C47, 75 (1990). R.M. Godbole and D.P. Roy, Phys. Rev. D43, 3640 (1990). R.M. Barnett et al., Phys. Rev. D47, 1048 (1993). J.F. Gunion and H. Haber, Nucl. Phys. B272, 1 (1986) (erratum: Nucl. Phys. B402, 567 (1993)); Nucl. Phys. B278, 449 (1986).

[4] See, for example, S. Dimopoulos, L.J. Hall and S. Raby, Phys. Rev. D45, 4192 (1992). V. Barger M.S. Berger and P. Ohmann, Phys. Rev. D47, 1093 (1993).

[5] G. Rodolfi, G. Ross and F. Zwirner, proceedings of the ECFA-LHC Workshop, CERN Report 90-10 (1990), Vol. II, p. 608. V. Bagger, S. Dimopoulos and E. Masso, Phys. Rev. Lett. 55, 920 (1985)

[6] J.F. Gunion et al., The Higgs hunter's guide (Addison-Wesley, Redwood City CA, 1990) and references therein.

[7] M. Drees and D.P. Roy, Phys. Lett. B269, 155 (1991)

[8] S. Moretti, and W.J. Stirling, Phys. Lett. B347, 291 (1995) (erratum: Phys. Lett. B366, 451 (1996)). E. Barradas et al., Phys. Rev. D53, 1678 (1996). A. Djouadi, J. Kalinowski, and P.M. Zerwas, Z. Phys. C70, 435 (1996). Related work in the $H^{+} \rightarrow W^{+} \Upsilon$ mode was done by J.A. Grifols, J.F. Gunion and A. Mendez, Phys. Lett. B197, 266 (1987); R.W. Robinett and L. Weinkauf, Mod. Phys. Lett. A6, 1575 (1991).

[9] V. Barger and R.J.N. Phillips, Collider Physics (Addison-Wesley, Redwood City CA, 1987). 
[10] J.A. Grifols and A. Mendez, Phys. Rev. D22, 1725 (1980).

[11] M. Guchait and D.P. Roy, Phys. Rev. D55, 7263 (1997)

[12] F. Abe et al. (CDF Collaboration), Phys. Rev. Lett. 79, 357 (1997).

[13] S. Raychaudhuri and D.P. Roy, Phys. Rev. D52, 1556 (1995); Phys. Rev. D53, 4902 (1996).

[14] S. Abachi et al. (DØ Collaboration), Phys. Rev. Lett. 79, 1203 (1997). F. Abe et al. (CDF Collaboration), hep-ex/9710008 (unpublished).

[15] S. Catani et al., Phys. Lett. B378, 329 (1996). E. Berger and H. Contopanagos, Phys. Rev. D54, 3085 (1996).

[16] E. Keith, E. Ma and D.P. Roy, Phys. Rev. D56, R5306 (1997).

[17] P. Janot, proceedings of the European Physics Conference, Jerusalem, Israel (1997)

[18] Private communications from G.P. Yeh and M. Mangano.

[19] F. Abe et al. (CDF Collaboration), Phys. Rev. Lett. 79, 3819 (1997). 